\title{
Factors Influencing an Eruption of Teeth Associated with a Dentigerous Cyst. A Systematic Review
}

\author{
Marek Nahajowski ( Marek_Nahajowski@wp.pl) \\ Department of Dentofacial Orthopedics and Orthodontics, Wroclaw Medical University. Krakowska 26, 50-425 Wroclaw, Poland. \\ https://orcid.org/0000-0003-4679-5649 \\ Sylwia Hnitecka \\ Opole University: Uniwersytet Opolski \\ Michał Sarul \\ Wroclaw Medical University: Uniwersytet Medyczny im Piastow Slaskich we Wroclawiu \\ Kornelia Rumin \\ Wroclaw Medical University: Uniwersytet Medyczny im Piastow Slaskich we Wroclawiu \\ Magdalena Dubowik \\ Wroclaw Medical University: Uniwersytet Medyczny im Piastow Slaskich we Wroclawiu \\ Joanna Antoszewska-Smith \\ Wroclaw Medical University: Uniwersytet Medyczny im Piastow Slaskich we Wroclawiu
}

\section{Research article}

Keywords: systematic review, dentigerous cyst, eruption interval, orthodontics

Posted Date: October 19th, 2020

DOI: https://doi.org/10.21203/rs.3.rs-92689/v1

License: (ㄷ) (i) This work is licensed under a Creative Commons Attribution 4.0 International License. Read Full License

Version of Record: A version of this preprint was published at BMC Oral Health on April 7th, 2021. See the published version at https://doi.org/10.1186/s12903-021-01542-y. 


\section{Abstract}

Aim

This systematic review aimed to identify factors conducive/inconducive to a spontaneous eruption of teeth after a dentigerous cyst's marsupialization.

Methods

In accordance with the PRISMA guidelines, the main research question was defined in the PICO format (P: patients with dentigerous cysts, I: spontaneous eruption after surgery, C: forced eruption, O: determining factors potentially influencing the intervention or the comparison). The MEDLINE, the EMBASE, and the Cochrane Central Register of Controlled Trials databases were searched for the keywords combining dentigerous/odontogenic/follicular cyst with teeth and/or orthodontics, as well as human teeth and eruption pattern/interval/period/duration. The qualified articles (4 out of 3005 found initially) provided following data: a rate of tooth eruption after surgical treatment of the cyst, along with age and gender of patients, a perpendicular projection distance between the top of the tooth cusp and the edge of the alveolar process, tooth angulation, a root formation stage, the cyst size, and space to erupt. The articles underwent analysis of the risk of bias and quality, with the ROBINS-I protocol and the modified Newcastle-Ottawa QAS, respectively. The systematic review was registered in PROSPERO under ID CRD42020189044.

Results

A real risk of bias was assessed as critical, while the quality of the studies was considered high (7-9 points in 9-point scale). Small distance between the top of a tooth cusp and the edge of the alveolar process, and space for eruption larger than tooth dimensions were the factors likely to favor spontaneous eruption. The cyst size was irrelevant, while the influence of the other factors could not be determined from the available data.

Conclusion

A small number of published studies, as well as their heterogeneity and the critical risk of bias do not allow creating the evidence-based protocol of management the teeth with DC after its marsupialization. More high-quality research is needed to be able to draw more reliable conclusions.

\section{Introduction}

According to a definition of the World Health Organization, a dentigerous cyst (DC), formerly known as a follicular one, is a pathology embracing the crown of a non-erupted tooth that attaches to its cementoenamel junction. It may transform into neoplastic compositions [1, 2]. It is the second most common bone cyst and affects $0.91-7.3 \%$ of the population [3, 4]. It is often located near the lower third molars and near the upper canines, lower premolars, and upper third molars. It leads to a lack of the tooth in the oral cavity or a delay in eruption, often complicated by an incorrect tooth position in the dental arch $[5,6]$.

For this reason, in addition to the procedure of decompression/enucleation of the cyst, it is essential to provide a proper intervention in the case of the teeth whose eruption process has been disturbed. As for the wisdom teeth, they are usually removed together with a cyst. However, others - mainly canines in the maxilla and premolars in the mandible - should be maintained $n$ order to secure continuity of the dental arches [6-10]. In such cases, the literature provides two options: 1. observation of the tooth after surgical intervention and waiting for its spontaneous eruption, and only if this process is delayed: implementation of orthodontic treatment; 2. immediate loading the tooth with orthodontic force [11-19]. Regardless of many factors potentially influence a spontaneous eruption, thus forcing the choice of one or the other treatment protocol [13, 17, 20-23], this issue has not been statistically assessed in a systematic review so far.

The study aimed to determine whether the evaluation of conducive or inconducive factors for a spontaneous tooth eruption after surgical treatment of a cyst, given in the literature, allows for creating an evidence-based protocol of clinical intervention favoring management of the DC-associated teeth in the dental arch.

\section{Materials And Methods}

The systematic review was registered in PROSPERO under ID CRD42020189044. The study was performed in accordance with the PRISMA (Preferred Reporting Items for Systematic Reviews and Meta-Analyses) guidelines, and the main research question was defined in the PICO (Population, Intervention, Comparison, Outcome) format. This way, a population $(P)$ was determined, including patients with the teeth associated with dentigerous cysts. A spontaneous tooth eruption after surgical DC treatment was considered an intervention (I), whereas a comparison (C) was the lack of a spontaneous tooth eruption after surgical DC treatment, i.e., the need for orthodontic traction. It was expected that an outcome of the study ( 0 ) would be a discovery of factors potentially influencing an intervention or comparison.

For the paper's purpose, the following electronic databases were searched: the PubMed, the EMBASE, and the Cochrane Central Register of Controlled Trials by entering the following keywords:

- dentigerous cyst and teeth,

- teeth and odontogenic cyst, 
- follicular cyst and teeth,

- follicular cyst and orthodontics,

- dentigerous cyst and orthodontics,

- odontogenic cyst and orthodontics,

- dentigerous cyst and teeth and orthodontics,

- teeth and odontogenic cyst and orthodontics,

- follicular cyst and teeth and orthodontics,

- human teeth and eruption pattern,

- human teeth and eruption timing,

- human teeth and eruption interval,

- human teeth and eruption period,

- human teeth and eruption duration.

Entering the keywords allowed retrieval of 3005 abstracts, which were then checked to see if they met the inclusion criteria: randomized clinical trials (RCT), prospective controlled clinical trials (CCT), case series, observational studies, review articles, and retrospective studies of generally healthy patients with DC-associated teeth. Single case reports, studies with limited data including conference abstracts and journal letters, studies of nonrepresentative groups, studies of patients with syndromes, and animal studies were rejected. Among the selected full-text articles, those written in a language other than English and not related to the current systematic review subject were rejected. In the remaining articles, bibliographies were reviewed. The following journals were manually screened: the American Journal of Orthodontics and Dentofacial Orthopedics, the International Orthodontics, the Journal of Clinical Orthodontics, the Journal of Oral and Maxillofacial Surgery, the Journal of Maxillofacial and Oral Surgery, the Journal of Stomatology, Oral and Maxillofacial Surgery, the BMC Oral Health, the Journal of Clinical Pediatric Dentistry, the Journal of Clinical and Experimental Dentistry, and the Journal of Dentistry for Children.

No article describing RCT or CCT results was found, nor were there any literature reviews on the permanent tooth eruption's physiological pace. There were only a few clinical studies and articles on the patients' mean age at the time of eruption of subsequent teeth, without specifying the process's duration. Six papers were qualified for the systematic review, two of which were rejected. A complete selection process is shown in Fig. 1.

From the four analyzed articles, we extracted: a sample size, a year of publication, a time elapsed from surgical treatment of the cyst to the tooth eruption, as well as the data potentially affecting the eruption process, such as patients' age and gender, a root formation stage, a perpendicularly measured distance from the alveolar ridge to the top of a tooth cusp, tooth angulation, cyst size, and eruption space.

An analysis of the risk of bias in individual articles was performed using the ROBINS-I (Risk of Bias In Non-randomized Studies of Interventions) tool, assessing three domains: 1. pre-intervention (bias due to confounding, the bias in the selection of participants into the study), 2. at-intervention (bias in classification of interventions) and 3. post-intervention (bias due to deviations from intended interventions, bias due to missing data, the bias in the measurement of outcomes and bias in the selection of the reported results), and then determining risks of bias for each of the articles equal to the lowest score awarded in all criteria. The risk of bias in all four studies was assessed as critical.

For a qualitative assessment, a modified Newcastle-Ottawa Quality Assessment Scale (QAS) was used, analyzing:

1) Patients' selection that is: A. representativeness of the group exposed to the tested factor, B. selection of patients for the control group, C. providing the source of data about individual patients, and D. demonstrating that the effects did not occur at the beginning of the study. For each positively assessed aspect, a maximum of 1 point was awarded.

2) The presence of confounding factors caused the control group to not affect the results in the same way as the study group. In this category, 0 to 2 points were awarded, depending on the significance of the influence of confounding factors.

3) Outcomes affected by: A.) blinding of evaluators, B.) follow-up duration longer than 12 months, and C.) Greater than 75 percent of patients who completed the study allowed for a maximum of 3 points.

\section{Results}

A direct comparison of results was possible due to the fact that all the studies concerned DC-associated lower premolars.

As for evaluating both intervention and the comparison, most of the teeth erupted spontaneously after marsupialization. However, some required orthodontic traction (Table 1). 
Table 1

Impact of Variables Defined in the Methodology on Teeth Eruption After DC Removal

\begin{tabular}{|c|c|c|c|c|c|c|c|c|}
\hline \multirow[b]{2}{*}{$\begin{array}{l}\text { Intervention and } \\
\text { Comparison }\end{array}$} & \multicolumn{2}{|c|}{ Fujii et al., 2008} & \multicolumn{2}{|c|}{ Hyomoto et al., 2003} & \multicolumn{2}{|c|}{ Qian et al., 2013} & \multicolumn{2}{|c|}{ Yahara et al., 2009} \\
\hline & $\begin{array}{l}\text { spontaneous } \\
\text { eruption }\end{array}$ & $\begin{array}{l}\text { lack of } \\
\text { spontaneous } \\
\text { eruption }\end{array}$ & $\begin{array}{l}\text { spontaneous } \\
\text { eruption }\end{array}$ & $\begin{array}{l}\text { lack of } \\
\text { spontaneous } \\
\text { eruption }\end{array}$ & $\begin{array}{l}\text { spontaneous } \\
\text { eruption }\end{array}$ & $\begin{array}{l}\text { lack of } \\
\text { physiological } \\
\text { eruption of } \\
\text { the opposite } \\
\text { teeth }\end{array}$ & $\begin{array}{l}\text { spontaneous } \\
\text { eruption }\end{array}$ & $\begin{array}{l}\text { lack of } \\
\text { spontaneous } \\
\text { eruption }\end{array}$ \\
\hline $\begin{array}{l}\text { Number of } \\
\text { teeth/number of } \\
\text { observations (n) }\end{array}$ & $45 / 60$ & $15 / 60$ & $38 / 47$ & $9 / 47$ & $15 / 15$ & $9 / 15$ & $15 / 21$ & $\begin{array}{l}6 / 21 \text { (no } \\
\text { eruption } \\
\text { after } 360 \\
\text { days) }\end{array}$ \\
\hline $\begin{array}{l}\text { Time elapsed } \\
\text { from } \\
\text { marsupialization } \\
\text { to orthodontic } \\
\text { force application }\end{array}$ & $x$ & NR & $x$ & NR & $\mathrm{x}$ & $\begin{array}{l}\text { no } \\
\text { orthodontic } \\
\text { treatment } \\
\text { performed }\end{array}$ & $x$ & NR \\
\hline $\begin{array}{l}\text { Eruption period } \\
\text { (days) }\end{array}$ & $90.4 \pm 91.8$ & $65.4 \pm 141.5$ & 109 & NR & $263 \pm 33$ & $807 \pm 112$ & $174 \pm 84$ & NR \\
\hline $\begin{array}{l}\text { Mean age } \\
\text { (years) }\end{array}$ & $10.5 \pm 1.9$ & $13.7 \pm 4.8$ & $10.6 \pm 2.0$ & $13.1 \pm 2.9$ & $9.1 \pm 0.8$ & $9.1 \pm 0.8$ & $9.8 \pm 2.1$ & $13.2 \pm 2.8$ \\
\hline $\operatorname{Sex}(n)$ & $33 \mathrm{M}, 27 \mathrm{~F}$ & & $\begin{array}{l}34 \mathrm{M}, 24 \mathrm{~F} \text { (wh } \\
\text { canines) }\end{array}$ & e group with & $7 \mathrm{M}, 4 \mathrm{~F}$ & & $12 \mathrm{M}, 9 \mathrm{~F}$ & \\
\hline $\begin{array}{l}\text { Cusp depth } \\
\text { (mm) }\end{array}$ & $4.5 \pm 4.3$ & $9.8 \pm 5.7$ & $4.4 \pm 4.5$ & $9.3 \pm 6.31$ & $5.4 \pm 5.2$ & $3.5 \pm 2.7$ & S: 14, D: 1 & S: $2, \mathrm{D}: 4^{1}$ \\
\hline Angulation $\left({ }^{\circ}\right)$ & $24.6 \pm 21.5$ & $52.7 \pm 45.0$ & $21.8 \pm 20.4$ & $67.7 \pm 49.8$ & $39.1 \pm 31.6$ & $10.5 \pm 8.3$ & $62.6 \pm 19.6$ & $26.6 \pm 21.7$ \\
\hline Root formation & NR & NR & $\begin{array}{l}<1 / 2: 17 \\
\text { 1/2-3/4: } 11, \\
3 / 4-4 / 4 \\
\text { (open apex): } \\
9, \text { mature } \\
\text { root: } 1\end{array}$ & $\begin{array}{l}<1 / 2: 3 \\
\text { 1/2-3/4: } 0 \text {, } \\
3 / 4-4 / 4 \\
\text { (open apex): } \\
\text { 2, mature } \\
\text { root: } 4\end{array}$ & $\begin{array}{l}<1 / 2: 7 \\
\text { 1/2-3/4: } 10\end{array}$ & $\begin{array}{l}<1 / 2: 6 \\
1 / 2-3 / 4: 7 \\
3 / 4-4 / 4: 4\end{array}$ & $\begin{array}{l}<1 / 2: 9,> \\
1 / 2: 6\end{array}$ & $\begin{array}{l}<1 / 2: 5,> \\
1 / 2: 1\end{array}$ \\
\hline Cyst size $\left(m m^{2}\right)$ & $\begin{array}{l}513.0 \pm \\
240.2\end{array}$ & $\begin{array}{l}465.1 \pm \\
344.2\end{array}$ & $\begin{array}{l}533.8 \pm \\
239.8\end{array}$ & $\begin{array}{l}546.7 \pm \\
406.5\end{array}$ & $\begin{array}{l}231.9 \pm \\
197.8\end{array}$ & no cyst & NR & \\
\hline Space/tooth size & $1.1 \pm 0.2$ & $0.8 \pm 0.6$ & $1.0 \pm 0.2$ & $1.0 \pm 0.5$ & $1.26 \pm 0.23$ & $1.28 \pm 0.24$ & $1.08 \pm 0.36$ & $1.14 \pm 0.12$ \\
\hline
\end{tabular}

Results of the analysis of the impact of variables defined in the methodology on tooth eruption after DC marsupialization are shown in Table 1. The risk of bias assessment is presented in Table 2, while the results of a qualitative assessment of articles based on QAS are given in Table 3.

Table 2

Risk of Bias

\begin{tabular}{|c|c|c|c|c|}
\hline & Study & & & \\
\hline Domain & Fujii et al. & Hyomoto et al. & Qian et al. & Yahara et al. \\
\hline Bias due to confounding & Serious risk & Serious risk & Serious risk & Serious risk \\
\hline Bias in the selection of participants into the study & Critical risk & Critical risk & Critical risk & Critical risk \\
\hline Bias in the classification of interventions & Serious risk & Serious risk & Serious risk & Serious risk \\
\hline Bias due to deviations from intended interventions & Low risk & Low risk & Low risk & Low risk \\
\hline Bias due to missing data & Low risk & Low risk & Low risk & Low risk \\
\hline Bias in measurement of outcomes & Moderate risk & Moderate risk & Moderate risk & Moderate risk \\
\hline Bias in the selection of the reported result & Moderate risk & Moderate risk & Moderate risk & Moderate risk \\
\hline Overall & Critical risk & Critical risk & Critical risk & Critical risk \\
\hline
\end{tabular}


Table 3

Quality Assessment According to the Modified Newcastle - Ottawa Scale

\begin{tabular}{|llll|}
\hline Study & Selection (0-4 points) & Comparability (0-2 points) & Outcome Assessment (0-3 points) \\
\hline Fujii et al. & 4 & 1 & 2 \\
\hline Hyomoto et al. & 4 & 1 & 2 \\
\hline Qian et al. & 4 & 2 & 3 \\
\hline Yahara et al. & 4 & 1 & 3 \\
\hline
\end{tabular}

\section{Discussion}

The dentigerous cyst is an interdisciplinary problem. Its development is usually asymptomatic for a long time. Delay in tooth eruption or its absence in the dental arch, or its incorrect position arouses anxiety. DC's consequences are a) bone destruction, b) root resorption, and sometimes when untreated, c) transformation into an odontogenic tumor (e.g., ameloblastoma) or a malignant tumor (e.g., squamous cell carcinoma) [1, 2]. The need to evacuate the cyst is obvious. However, preservation of the DC-associated teeth requires a reliable, not only intuitive protocol. Regardless of the fact that $81 \%$ of premolars may erupt spontaneously after surgical removal of the cyst or marsupialization/decompression [24], it does not exempt from continuous monitoring the factors conducive/inconducive to the eruption (both in terms of quantity and quality), which fully justifies the subject of our study.

Some authors claim that spontaneous tooth eruption should occur no later than three months after marsupialization; otherwise, orthodontic traction should be applied $[5,11,17]$. It is in accordance with the results of studies by Hyomoto et al. [21], where the teeth erupted within 109 days on average; after 110 days the number of erupting teeth evidently decreased. Duration of the spontaneous eruption was comparable or longer (5.8 \pm 2.8 months) in the study by Yahara et al. [23]. Here, the authors determined that if the tooth did not erupt at least halfway within 3-months post-surgery, it would most likely not reach the occlusal plane even within 12 months. Fujii et al. [20] and Hyomoto et al. [21] observed a longer duration of the spontaneous eruption, compared to the forced one (Table 1). This difference, however, was either statistically insignificant [20] or not analyzed [21]. In the patients studied by Qian et al. [22], all teeth erupted spontaneously after marsupialization, in a significantly shorter time than the teeth without DC ( $p=.001)$. It allowed the conclusion that marsupialization induces osteogenesis and the pressure reduction in the alveolar bone, which may stimulate faster eruption.

Nonetheless, such interpretation must be treated with caution; there are no reviews on a physiological pace of permanent tooth eruption, which one can compare with a DC-associated tooth eruption duration time.

The spontaneous eruption occurred mostly in patients within the first decade and the beginning of the second decade of their life. The average age of patients experiencing spontaneous eruption in almost all studies was significantly lower than the average age of patients whose eruption process was arrested (Table 1). Only Qian et al. [22] did not show a statistically significant age difference between the study and control groups, which is evident since they compared DC-associated teeth with those from the other quadrant in the same mandible.

The patient's age is closely related to the stage of root formation; therefore, these two factors may be analyzed simultaneously when assessing their impact on a spontaneous tooth eruption after marsupialization. In the study by Hyomoto et al., an initial stage of root formation $(r<1 / 2)$ increased the probability of spontaneous tooth eruption after marsupialization in a statistically significant manner $(p<.05)$. Nevertheless, other authors have not demonstrated such a relationship, proving that eruption is utterly independent of the degree of the root formation (Qian et al .: $p=.1 ;$ Yahara et al .: $p$ $=.6126$ ). Due to the fact that stages of root formation in the study and control groups differed from one article to another, the data is not entirely comparable.

It was impossible to determine the influence of patients' gender on the tooth eruption after marsupialization. The gender distribution in the study and control groups was established in none of the studies.

As for the distance between the top of a tooth cusp and the edge of the alveolar process in a perpendicular projection, Fujii et al. [20] observed significantly lower value in cases of spontaneous eruption after marsupialization than in cases of cystectomy or application of orthodontic traction ( $\mathrm{p}$ $<.0007)$. On this basis, the authors concluded that the tooth's position at a depth $<5.1 \mathrm{~mm}$ is one of the predictive parameters of a spontaneous eruption of a DC-associated tooth. Other authors have reached similar conclusions [21, 23]. In turn, Qian et al. [22] showed that despite shallower location of the teeth in the control group $(3.5 \pm 2.7)$, the duration time of their eruption was more prolonged, although it was not a statistically significant difference ( $p$ $=.137)$.

As for angulation of the teeth spontaneously erupted after marsupialization, in the study of Fujii et al. [20] and Hyomoto et al. [21], it was two and three times smaller on average, compared to angulation in cases requiring a cystectomy or orthodontic traction (Table 1). On this basis, Fujii et al. [20] determined that angulation $<25^{\circ}$ is one of the significant predictive parameters of DC-associated tooth eruption $(p=.0149)$. However, this conclusion was not confirmed in the work of Qian et al. [22] and Yahara et al. [23]; moreover, Qian et al. observed greater angulation values in the group of patients whose teeth erupted spontaneously.

The size of a dentigerous cyst was not found to be a predictive factor of a spontaneous eruption. However, in studies by Fujii et al. [20] and Hyomoto et al. [21], an average cyst size in cases where there was a spontaneous tooth eruption after marsupialization was similar, while Qian et al. [22] provided a

Page 5/9 
much lower value (Table 1). Based on these results, it can be concluded that from an orthodontic point of view, the discussed parameter is not a key one in treatment planning. However, it may have an impact on decisions regarding a surgical procedure.

In the work of Fujii et al. [20], the authors described a difference between groups as statistically significant $(p<.05)$, therefore concluding that a ratio greater than 1:1 is a factor conducive to a spontaneous eruption.

Risks of bias in a pre-intervention domain were considered serious in all works. The reasons were: 1. a failure to use interference elimination methods, such as stratification, regression, matching, standardization or inverse probability weighting, 2 . retrospective nature of the research, namely: forming study and control groups after the results have been achieved. Thus, prognostic variables might have been deliberately selected to confirm the assumed hypotheses and 3. selection of participants from a larger group of patients treated in a given unit in a specific timeline, which increases the probability of disturbing the proportion of a primary cohort. This study did not adopt a critical risk because the study group's inclusion criteria were precisely defined in all studies.

Since there was no misclassification of an intervention status, the intervention domain's risk was assessed as low. The study participants were treated routinely, using standard procedures, and medical records were kept. Ongoing records were available entirely to the authors; therefore, a risk of bias due to deviations from the intended interventions and risks of bias due to missing data were considered low. The risks of bias in the measurement of outcomes were defined as moderate because methods of assessing the results were objective and carried out in the same way for the study and control groups. Nevertheless, the lack of randomization made the measurement results potentially dependent on whether the patient belonged to the study or control group.

The risk of bias in selecting the reported results was also moderate because the authors provided all the results without using the data obtained in previous analyzes, despite the studies' retrospective nature.

The risk of bias in all four studies was considered critical. However, despite serious risks of bias of all qualified works, it must not be forgotten that serious risks were recorded only in the pre-intervention domain. The reason was a retrospective nature of the study, which is an obvious disadvantage, however easy to overcome in future studies on this aspect.

As for the qualitative assessment based on QAS, in the "Patient Selection" category, the work by Fujii et al. [20], Hyomoto et al. [21], and Yahara et al. [23] obtained 4 points because the patients were appropriately classified into groups according to a differentiating factor, which was either a spontaneous eruption or its lack after marsupialization. Moreover, there was full documentation available, proving that the studied effects did not occur at the beginning of the research. The article by Qian et al. [22] lost 0.5 points for low representativeness of the study group and another 0.5 points due to the qualitatively weaker control group, which were the participants' healthy teeth from the study group. In turn, such construction of study and control groups by Qian et al. provided no additional differentiating factors. Therefore, this paper awarded 2 points in the section on confounding factors. Articles by Fujii et al. [20] and Hyomoto et al. [21] lost 1 point due to age differences between the control and study groups, while the work of Yahara et al. [23] did not receive points in this category due to significant differences in the size of the control and study groups, which could be a significant factor affecting the studied variables. In the effect assessment section, all articles lost 1 point each due to a lack of evaluators' blinding. The works of Fujii et al. [20] and Hyomoto et al. [21] lost 1 additional point due to applying orthodontic traction sooner than three months after marsupialization or performing cystectomy, which could have influenced an assessment of time needed for a tooth to erupt spontaneously.

\section{Limitations}

In this systematic review, the most important database available was searched through using a comprehensive search strategy. However, non-English searches were not undertaken, posing a risk of incomplete yield, which could have had an impact on the obtained results due to the publication bias.

So far, very little research has been conducted on the management of the DC-associated teeth. Apart from merely four retrospective works, which have been selected, only case reports and case series are available [7-19, 25,26], where the authors present selected methods of treatment, based on individual clinical experiences or protocols adopted in a given unit, which is not in line with the evidence-based medicine.

\section{Conclusion}

A small number of published studies, as well as differences between available studies in terms of size of the groups, their characteristics depending on age, gender, and period of observation, do not allow to provide a list of objective criteria allowing for making decisions about the implementation or withdrawal of orthodontic traction after marsupialization. A small distance between the top of a tooth cusp and the edge of the alveolar process and the space for eruption larger than tooth dimensions are the factors, which increase the probability of a spontaneous eruption. Determining the influence of the remaining factors requires further research, primarily with the same inclusion criteria and homogeneous groups, which would enable the development of the reliable and reproducible protocol of management of the DC-associated teeth, much needed in everyday clinical practice.

\section{Abbreviations}

DC - dentigerous cyst 
PRISMA - Preferred Reporting Items for Systematic Reviews and Meta-Analyses

PICO - Population, Intervention, Comparison, Outcome

RCT - randomized clinical trial

CCT - controlled clinical trial

ROBINS-I - Risk of Bias In Non-randomized Studies of Interventions

QAS - Quality Assessment Scale

\section{Declarations}

\section{Ethics Approval and Consent to Participate:}

not applicable

\section{Consent for Publication:}

not applicable

\section{Availability of Data and Materials:}

the datasets used and/or analyzed during the current study are available from the corresponding author on reasonable request.

\section{Competing Interests:}

The authors declare that they have no competing interests

\section{Funding:}

none

\section{Authors' Contributions:}

$\mathrm{MN}$ and SH collected the data and were major contributors in writing the manuscript. MN, SH, and JAS analyzed and interpreted the data. MS, KR and MD created the tables and prepared the references list. All the authors performed the critical revision and final approval of the article.

\section{Acknowledgments:}

not applicable

\section{References}

1. Bilodeau EA, Collins BM. Odontogenic Cysts and Neoplasms. Surg Pathol Clin. 2017; 10:177-222.

2. Nayyer NV, Macluskey M, Keys W. Odontogenic Cysts - An Overview. Dent Update. 2015; 42:548-55.

3. Açikgöz A, Uzun-Bulut E, Özden B, Gündüz K. Prevalence and distribution of odontogenic and nonodontogenic cysts in a Turkish population. Med Oral Patol Oral Cir Bucal. 2012;17:e108-e115.

4. Johnson NR, Gannon OM, Savage NW, Batstone MD. Frequency of odontogenic cysts and tumors: a systematic review. J Investig Clin Dent. 2014; 5:9-14.

5. Contar CM, Thomé CA, Pompermayer A, Sarot JR, Vinagre RO, Machado MÂ. Marsupialization of dentigerous cyst: report of a case. J Maxillofac Oral Surg. 2015; 14:4-6.

6. Rumin K, Rumin-Wojciechowska A, Zając K, Antoszewska J, Sarul M. Management of impacted and displaced teeth due to odontogenic cysts in growing patients. Clinical Orthodontics 2017; 3:7-22.

7. Berdén J, Koch G, Ullbro C. Case series: Treatment of large dentigerous cysts in children. Eur Arch Paediatr Dent. 2010;11:140-5. 
8. Kirtaniya BC, Sachdev V, Singla A, Sharma AK. Marsupialization: a conservative approach for treating dentigerous cyst in children in the mixed dentition. J Indian Soc Pedod Prev Dent. 2010;28:203-8.

9. Serra e Silva FM, Sawazaki R, de Moraes M. Eruption of teeth associated with a dentigerous cyst by only marsupialization treatment: a case report. J Dent Child (Chic). 2007;74:228-230.

10. Yücel Ö, Yildirim G, Tosun G, Müge Baka Z, Bedii Göyenç Y, Günhan Ö. Eruption of impacted permanent teeth after treatment of a dentigerous cyst: a case report. J Dent Child (Chic). 2013;80:92-6.

11. Aoki N, Ise K, Inoue A, et al. Multidisciplinary approach for treatment of a dentigerous cyst - marsupialization, orthodontic treatment, and implant placement: a case report. J Med Case Rep. 2018;12:305-11.

12. Ertas U, Yavuz MS. Interesting eruption of 4 teeth associated with a large dentigerous cyst in mandible by only marsupialization. $\mathrm{J}$ Oral Maxillofac Surg. 2003;61:728-30.

13. Gurler G, Yilmaz S, Delilbasi C, Dilaver E, Yuzbasioglu E, Patir-Munevveroglu A. Conservative surgical treatment of the jaw cysts in children: Case study of five patients. Niger J Clin Pract. 2017;20:1216-20.

14. Jena AK, Duggal R, Roychoudhury A, Parkash H. Orthodontic assisted tooth eruption in a dentigerous cyst: a case report. J Clin Pediatr Dent. 2004;29:33-5.

15. Koca H, Esin A, Aycan K. Outcome of dentigerous cysts treated with marsupialization. J Clin Pediatr Dent. 2009;34:165-8.

16. Kumar Mohapatra P, Joshi N. Conservative management of a dentigerous cyst associated with an impacted mandibular second premolar in mixed. J Dent Res Dent Clin Dent Prospects. 2009;3:98-102.

17. Miyawaki S, Hyomoto M, Tsubouchi J, Kirita T, Sugimura M. Eruption speed and rate of angulation change of a cyst-associated mandibular second premolar after marsupialization of a dentigerous cyst. Am J Orthod Dentofacial Orthop. 1999;116:578-84.

18. Sain DR, Hollis WA, Togrye AR. Correction of a superiorly displaced impacted canine due to a large dentigerous cyst. Am J Orthod Dentofacial Orthop. 1992;102:270-6.

19. Takagi S, Koyama S. Guided eruption of an impacted second premolar associated with a dentigerous cyst in the maxillary sinus of a 6 -year-old child. J Oral Maxillofac Surg. 1998;56:237-9.

20. Fujii R, Kawakami M, Hyomoto M, Ishida J, Kirita T. Panoramic findings for predicting eruption of mandibular premolars associated with dentigerous cyst after marsupialization. J Oral Maxillofac Surg. 2008;66:272-6.

21. Hyomoto M, Kawakami M, Inoue M, Kirita T. Clinical conditions for eruption of maxillary canines and mandibular premolars associated with dentigerous cysts. Am J Orthod Dentofacial Orthop. 2003;124:515-520.

22. Qian WT, Ma ZG, Xie QY, Cai XY, Zhang Y, Yang C. Marsupialization facilitates eruption of dentigerous cyst-associated mandibular premolars in preadolescent patients. J Oral Maxillofac Surg. 2013;71:1825-32.

23. Yahara Y, Kubota Y, Yamashiro T, Shirasuna K. Eruption prediction of mandibular premolars associated with dentigerous cysts. Oral Surg Oral Med Oral Pathol Oral Radiol Endod. 2009;108:28-31.

24. Kaczmarzyk T. Torbiele obszaru szczękowo-twarzowego. Warszawa: Kwintesencja; 2015.

25. Ishihara Y, Kamioka H, Takano-Yamamoto T, Yamashiro T. Patient with nonsyndromic bilateral and multiple impacted teeth and dentigerous cysts. Am J Orthod Dentofacial Orthop. 2012;141:228-41.

26. Maltoni I, Santucci G, Maltoni M, Zoli L, Perri A, Gracco A. Recovering teeth from a large dentigerous cyst: A case report. Int Orthod. 2015;13:232-44.

\section{Figures}




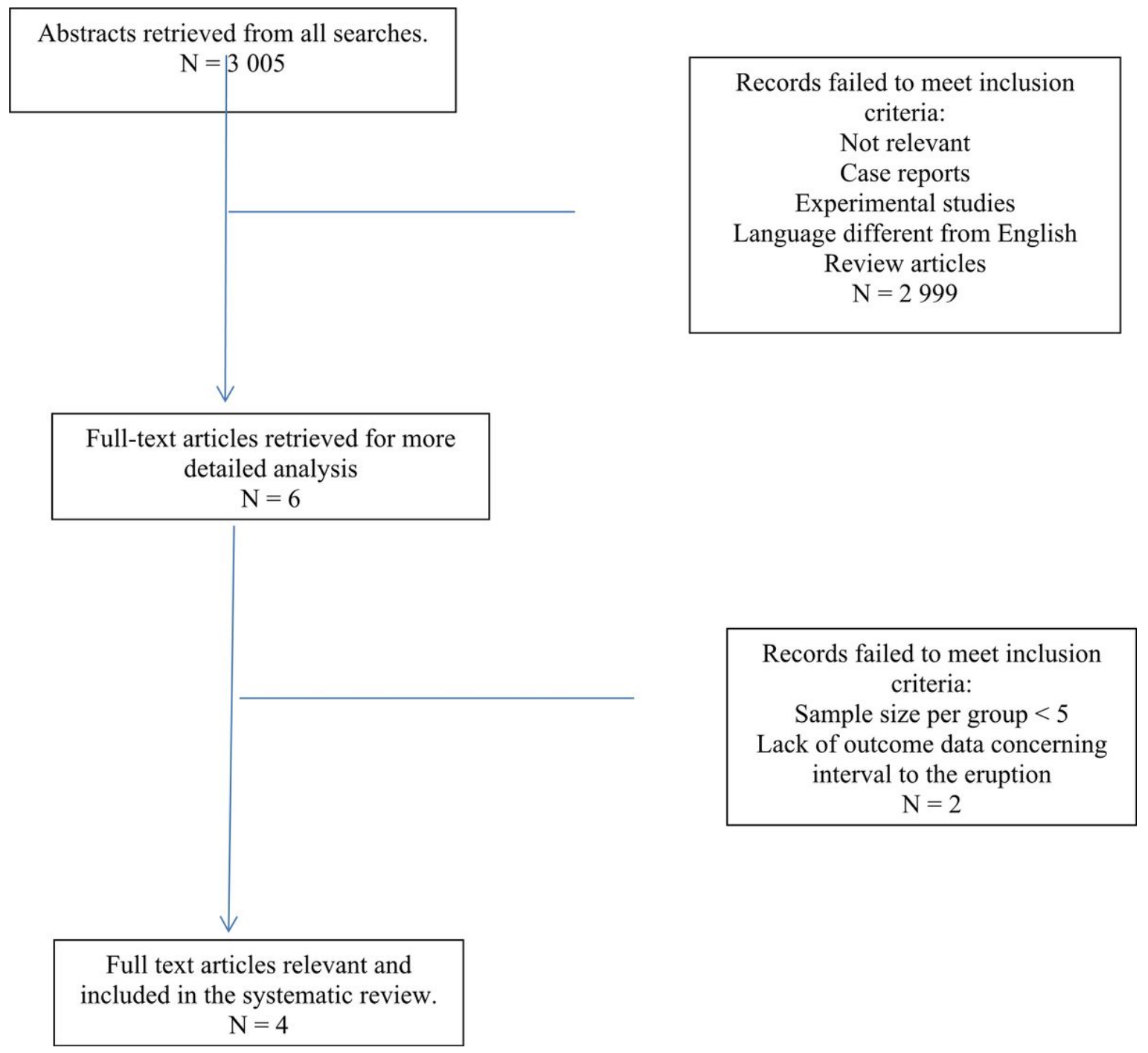

Figure 1

Flow chart

\section{Supplementary Files}

This is a list of supplementary files associated with this preprint. Click to download.

- PRISMAchecklist.doc 